Virginia Commonwealth University vCU Scholars Compass

2016

\title{
Tuning the plasmonic properties of silver nanopatterns fabricated by shadow nanosphere lithography
}

Whitney Ingram

University of Georgia

Yizhuo He

University of Georgia

Keenan Stone

University of Georgia

See next page for additional authors

Follow this and additional works at: http://scholarscompass.vcu.edu/phys_pubs

Part of the Physics Commons

(C) 2016 IOP Publishing Ltd

\section{Downloaded from}

http://scholarscompass.vcu.edu/phys_pubs/207

This Article is brought to you for free and open access by the Dept. of Physics at VCU Scholars Compass. It has been accepted for inclusion in Physics Publications by an authorized administrator of VCU Scholars Compass. For more information, please contact libcompass@vcu.edu. 
Authors

Whitney Ingram, Yizhuo He, Keenan Stone, William M. Dennis, Dexian Ye, and Yiping Zhao 


\title{
Tuning the plasmonic properties of silver nanopatterns fabricated by shadow nanosphere lithography
}

\author{
Whitney Ingram $^{1}$, Yizhuo He ${ }^{1}$, Keenan Stone ${ }^{1}$, William M Dennis ${ }^{1}$, \\ Dexian $\mathrm{Ye}^{2}$ and Yiping Zhao' \\ ${ }^{1}$ Department of Physics and Astronomy, and Nanoscale Science and Engineering Center, University of \\ Georgia, Athens, GA 30602, USA \\ ${ }^{2}$ Department of Physics, Virginia Commonwealth University, Richmond, VA 23284, USA \\ E-mail: wmi613@uga.edu
}

Received 16 March 2016, revised 13 July 2016

Accepted for publication 25 July 2016

Published 16 August 2016

\begin{abstract}
Regular silver $(\mathrm{Ag})$ nanopatterns, from disconnected nanotriangles to well coupled triangular clusters of nanoparticles, were prepared by shadow nanosphere lithography at different incident angles $\theta$ from $0^{\circ}$ to $20^{\circ}$ with continuous azimuthal rotation. The resulting nanopatterns were consistent with predictions by numerical calculations and Monte Carlo simulations of adatoms with high diffusivity. The visible localized surface plasmon resonance of these nanopatterns was tuned by $\theta$ systematically due to the change in size, shape, and arrangement of Ag nanopatterns. These resonances were consistent with finite-difference time-domain simulations using realistic nanopatterns based upon scanning electron micrographs. Such a simple fabrication strategy can be used to optimize surface enhanced Raman scattering substrate fabrication, as well as other plasmonics based applications.
\end{abstract}

S Online supplementary data available from stacks.iop.org/NANO/27/385301/mmedia

Keywords: shadow nanosphere lithography, finite-difference time-domain, Monte Carlo simulation, surface enhanced Raman spectroscopy, localized surface plasmon resonance

(Some figures may appear in colour only in the online journal)

\section{Introduction}

The fabrication and advancement of plasmonic nanostructures have many interesting and important applications, such as metamaterials [1, 2], solar energy conversion [3], ultra-sensitive chemical and biological sensing [4, 5], plasmonic heating [6, 7], and surface enhanced Raman spectroscopy (SERS) [8, 9]. One popular method to prepare plasmonic nanostructures is by nanosphere lithography (NSL). This method combines a self-assembled monolayer or multilayer of nanospheres with a physical vapor deposition technique to produce a large variety of nanopatterns including nanorings, dots, grids, wires, etc, with control over the separation distance and material of the nanopatterns [10-13]. Unlike photolithography and electron beam lithography, NSL is considered a cost-effective and scalable technique, which is ideal for practical applications.

The plasmonic properties of nanopatterns generated by NSL can be tuned using two different strategies. The first strategy is based on changing the colloidal monolayer template. This work was first performed by Van Duyne et al, and they demonstrated that the localized surface plasmon resonance (LSPR) of triangular nanopatterns could be tuned by changing the colloid size, deposition thickness, as well as altering their shape by annealing them [14]. From the same research group Haynes et al showed that the LSPR of Ag nanopatterns could be changed by stacking two monolayer templates together to obtain circular shaped Ag nanopatterns [15]. The second strategy, referred to as angle-resolved or shadow nanosphere lithography (SNSL), can also be used to tune the LSPR by a fixed colloidal monolayer $[16,17]$. SNSL 
is the manipulation of the position or the orientation of the colloid template with respect to incident vapor direction during a deposition to produce a variety of nanopatterns. Van Duyne et al also pioneered this strategy by controlling the vapor incident angle, $\theta$, defined as the angle between the normal of the substrate and vapor source, to fabricate nanosized triangular patterns [18]. In general, nanopatterns fabricated by SNSL techniques are performed by controlling material vapor flux direction in four different ways: fixed $\theta$, stepwise change in $\theta$, azimuthal rotation, or phi-sweep of the substrate. To create more complex patterns, the colloid monolayer template may be modified by reactive ion etching (RIE) or annealing. For instance, Kosiorek et al first annealed the colloid monolayers, then rotated the templated substrate during the deposition to produce nanorod, dot, and nanoring arrays [19]. Similarly, Gwinner et al annealed polystyrene (PS) nanospheres, then deposited $\mathrm{Ag}$ at $\theta$ between $15^{\circ}$ and $20^{\circ}$ while rotating and sweeping azimuthal angles to produce nanorings with complete and incomplete rotations [20]. Stepwise nanopatterns were first demonstrated by Zhang et al, and showed that colloid nanopatterns first etched by RIE, then deposited with stepwise change of both $\theta$ and azimuthal position $\varphi$ to produce complex quasi 3D grids [21]. More recently, Nemiroski et al also combined SNSL and RIE to produce complex assemblies of nanopatterned metasurfaces with both single and multiple materials [22]. Similarly, Chen et al prepared gold ( $\mathrm{Au}$ ) Moiré patterns by etching double layered colloid templates [23]. Thus, SNSL is a powerful method to tune the plasmonic properties of metal nanopatterns.

Here we will show another variation of SNSL. Using SNSL with a continuous azimuthal substrate rotation, different nanopatterns can be formed with tunable LSPR properties, which can be used for SERS substrate fabrication optimization or other plasmonic applications.

\section{Experimental details}

\section{Materials}

500 nm diameter PS nanospheres (Polyscience, Lot \# 07107) were used to form the colloid monolayers onto cleaned glass slides (Gold Seal, Part \# 3010) and silicon wafers (University Wafer). Ethanol (Sigma-Aldrich, 98\%), toluene (Fisher Scientific, 99.8\%), acetone (Fisher-Scientific, 99.8\%), and 2-propanol (Fisher-Scientific, 99.8\%) were used for colloid monolayer preparation and to remove residual PS from substrates after Ag deposition. Sulfuric acid (Fisher Scientific, 98\%), ammonium hydroxide (Fisher Scientific, 98\%), and hydrogen peroxide (Fisher Scientific, 30\%) were used without further purification. Silver pellets $(99.999 \%)$ were purchased from Kurt J Lesker company. 1,2-Di(4-pyridyl)ethylene (BPE) (Sigma-Aldrich, 99\%) diluted in methanol (FisherScientific, 99\%) was prepared as an analyte for SERS measurements. De-ionized (DI) water $(18 \mathrm{M} \Omega \mathrm{cm})$ was used throughout the experiments.

\section{Substrate preparation}

Prior to monolayer self-assembly, glass and silicon were cut into $1.2 \times 1.2 \mathrm{~cm}^{2}$ pieces. Glass substrates were cleaned in a boiling piranha solution $(4: 1 \mathrm{v} / \mathrm{v}$ of sulfuric acid: hydrogen peroxide) for at least $20 \mathrm{~min}$. Silicon pieces were cleaned using the RCA-1 cleaning method $(5: 1: 1 \mathrm{v} / \mathrm{v} / \mathrm{v}$ of water: ammonium hydroxide: hydrogen peroxide) at $\sim 70{ }^{\circ} \mathrm{C}$ for at least $20 \mathrm{~min}$. After chemical cleaning, all substrates were thoroughly rinsed in DI water and dried with $\mathrm{N}_{2}$ gas.

\section{Colloid monolayer formation}

Colloid monolayers were assembled using an air/water interface technique as previously reported [24, 25]. Briefly, the PS nanosphere suspension, purchased initially at a concentration of $2.6 \mathrm{wt} . \%$ in water, was diluted to $0.4 \mathrm{wt} . \%$ in DI water, and further diluted with ethanol to a $2: 1$ volumetric ratio. The suspension was loaded into a syringe and mounted onto a syringe pump (KD Scientific). A cleaned glass Petri dish (diameter of $10 \mathrm{~cm}$ ), pre-filled with $24 \mathrm{ml}$ of DI water, was tilted at an angle of $\sim 7^{\circ}$, and a needle attached to the syringe was bent towards the water within the Petri dish. Droplets of PS solution were dispensed onto the water surface at a rate of $0.015 \mathrm{ml} \mathrm{min}^{-1}$. Each drop spread radially outward from the droplet location, and a monolayer of PS nanospheres was slowly formed on the water surface along the edge of the Petri dish. This process continued until a monolayer of PS nanospheres covered nearly the entire water surface. A Teflon ring, with a diameter smaller than the diameter of the Petri dish, was placed gently on the water surface to protect the monolayer film against adhering to the side wall of the Petri dish. After the water level was raised, glass and silicon pieces were slid carefully under the water to the area below the monolayer film. Finally, the monolayer film was deposited onto the surface of the submerged substrates by slowly pumping water out from the Petri dish.

\section{Ag nanopattern fabrication}

Ag nanopatterns were prepared using a custom-built electron beam deposition system with a deposition configuration shown in (figure 1). Monolayer coated substrates were mounted on a holder placed $50.8 \mathrm{~cm}$ above the crucible. Depositions were performed when the pressure reached $10^{-6}$ Torr. Ag was deposited at a deposition rate of $0.1 \mathrm{~nm} \mathrm{~s}^{-1}$, which was monitored by a quartz crystal microbalance facing the source. As shown in (figure 1), the monolayer coated substrates were positioned at a fixed angle $\theta$ formed between the substrate surface normal and the vapor deposition direction, and were rotated azimuthally at a speed of $20 \mathrm{rpm}$ for the duration of the deposition. For each deposition, $\theta$ was set to $0^{\circ}, 5^{\circ}, 7^{\circ}, 10^{\circ}, 12^{\circ}, 15^{\circ}, 17^{\circ}$, and $20^{\circ}$, respectively, with an accuracy of $\pm 1^{\circ}$. For different $\theta$, the deposited Ag thickness were initially varied to ensure that the final thickness of the Ag patterns was $\sim 50 \mathrm{~nm}$, which was determined by atomic force microscope (AFM) measurements. However, as $\theta$ increased, the deposition made the shadowing effect of the opening among nanospheres smaller 


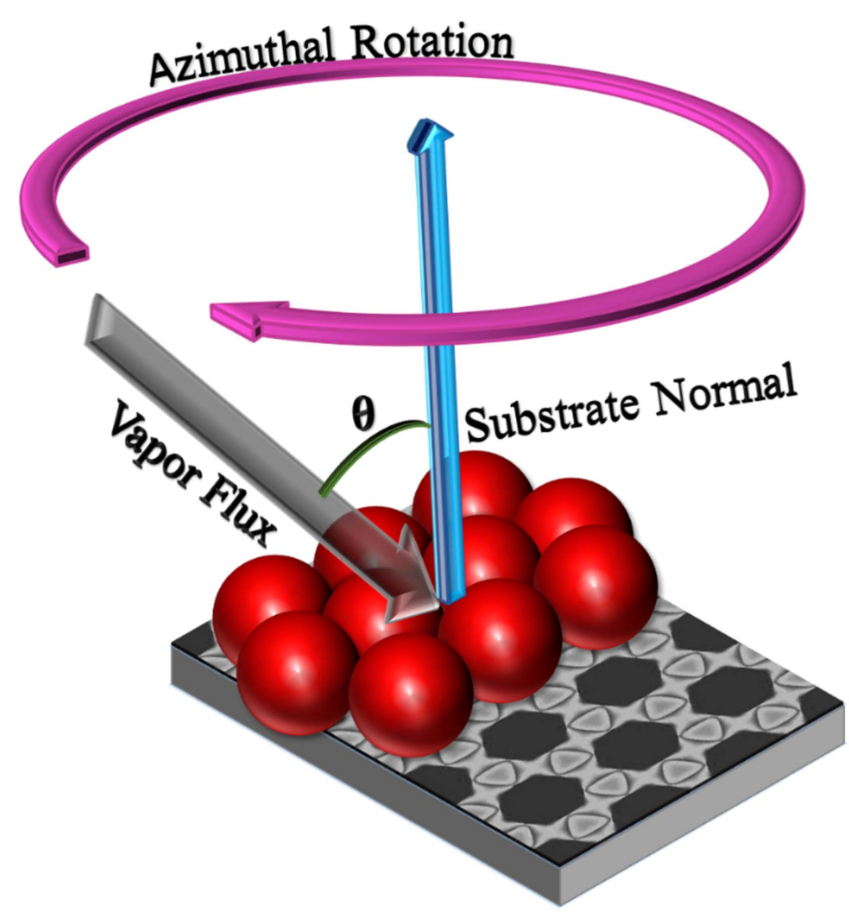

Figure 1. Schematic diagram of shadow nanosphere lithography (SNSL).

and smaller, which minimized the actual Ag film thickness. Therefore, the Ag nanopatterns possess decreased thickness with increasing $\theta$. After the Ag deposition, the monolayer template was removed using Scotch tape, and then the substrates were rinsed in toluene, acetone and 2-propanol successively to remove PS residual.

\section{Substrate characterization}

The optical transmission spectra of the Ag nanopatterns were measured by an ultraviolet-visible spectrophotometer (UVvis, Jasco-750) at four different locations. The optical reflectance spectra were measured by a home-built reflectance measurement with set-up details described in [26]. Due to the low reflectivity of the Ag nanopatterns (see figure S3.2 of supporting information), the transmission spectra of the $\mathrm{Ag}$ nanopatterns could be converted to extinction $(E)$ by the following equation: $E=-\ln T$. SERS spectra of the $\mathrm{Ag}$ nanopatterns were measured using a confocal Raman microscope (Renishaw inVia), with an excitation wavelength of $633 \mathrm{~nm}$. A $2 \mu \mathrm{l}$ droplet of BPE $\left(10^{-4} \mathrm{M}\right)$ in methanol was dispensed onto the Ag nanopattern substrate. The nine SERS spectra measured from randomly chosen positions on each substrate were obtained. All quantitative analysis of the average SERS spectra, such as peak positon and amplitude, were processed using the spectroscopic software GRAMS AI (Thermo Scientific).

\section{Numerical calculations (NCs) and Monte Carlo (MC) simulations}

An in-house Matlab program (previously reported) was used to calculate the $\mathrm{Ag}$ nanopattern formation at different $\theta$. This calculation predicted the thickness distribution of the Ag film on the substrate by considering the shadowing effects from thirty-six nearest neighbor PS nanospheres. Detailed information can be found in [25].

The formation of $\mathrm{Ag}$ nanopatterns was also simulated by a kinetic, three-dimensional MC simulation. In the MC ballistic deposition model, the incident particles approached the surface in a straight trajectory at an angle of $\theta$. When the particle arrived on the surface or passed by the nearestneighbor site of a previously deposited particle, it would deposit and become part of the surface. In order to simulate the surface diffusion after the incoming particle settled on the surface, one particle on the surface would be randomly chosen to perform the diffusion. The diffusion was a random walk on the surface with a diffusion length of one unit. There was a predetermined number $D$ of particles selected to perform diffusion, which represented the strength of diffusivity of a particular material such as Ag. Pre-occupied sites above a planar surface were formed according to the hexagonal pattern of nanospheres to mimic the templates used in experiments. The substrate rotation was included in the MC simulation. After the deposition was done, the templates and the deposition above the top surface of the templates were digitally removed. Additional calculation details and assumptions are provided in supplementary information S1.

The optical properties of selected $\mathrm{Ag}$ nanopatterns deposited at $\theta=0^{\circ}, 10^{\circ}, 12^{\circ}, 17^{\circ}$, and $20^{\circ}$ were simulated using the finite-difference time-domain (FDTD) method $[27,28]$. The Ag nanopattern simulation models were based upon AFM data and SEM images. The details of the electromagnetic modeling process are given in supplementary information S2.

\section{Results and discussion}

\section{Morphology}

(Figures 2(a)-(c)) show representative Ag nanopatterns predicted by NCs, experimentally obtained via SEM, and generated by $\mathrm{MC}$ simulation for $\theta=0^{\circ}, 10^{\circ}, 15^{\circ}$, and $20^{\circ}$, respectively. SEM micrographs and simulation results for $\theta=5^{\circ}, 7^{\circ}, 12^{\circ}$, and $17^{\circ}$, are shown in supplementary information S3.1. As predicted by the NCs (figure 2(a)), when $\theta=0^{\circ}$, individual and disconnected nanotriangles were formed on the substrates. When $\theta$ was increased from $0^{\circ}$ to $10^{\circ}$, the characteristic pattern changed to a hexagonal shape with a thick central triangle (the red colored areas), while the neighboring patterns remained unattached. As $\theta$ changed to $15^{\circ}$ and $20^{\circ}$, each individual pattern started to join with the adjacent patterns, and individual pattern became a cup-like triangular shape, i.e., there was a triangular hole in the middle of each pattern. In general, as $\theta$ increased, the hole became larger, and the entire pattern grew larger as well. But the overall arrangement for all the patterns still followed closepacked symmetry regardless of the $\theta$ value. Notice that for $\theta \geqslant 40^{\circ}$, there were no patterns formed on the substrate due to the shadowing effect. These predictions matched well with 


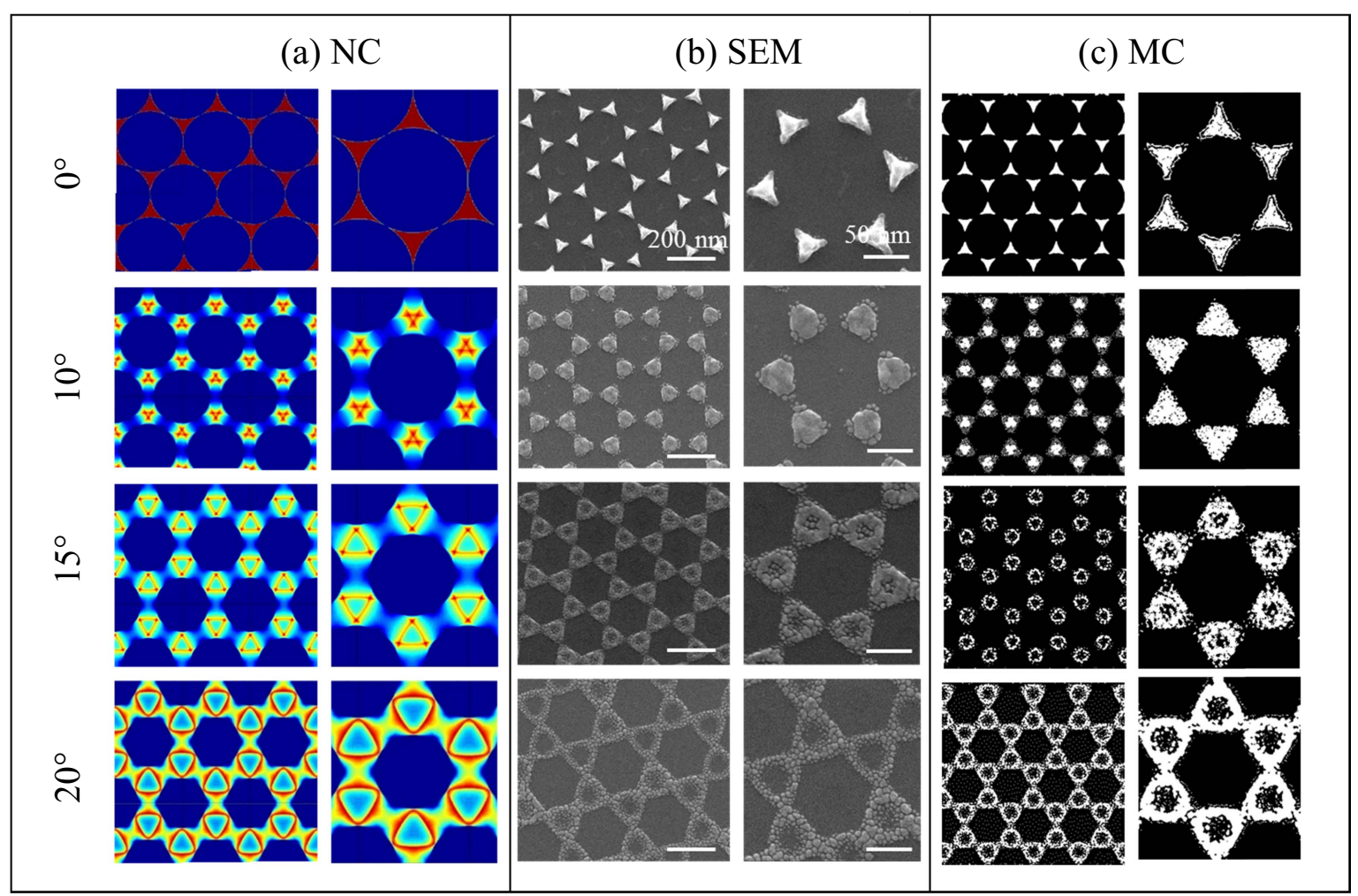

Figure 2. Representative nanopatterns formed by SNSL: (a) numerical calculations (NC), (b) experimental SEM images, and (c) Monte Carlo (MC) simulation at $\theta=0^{\circ}, 10^{\circ}, 15^{\circ}$, and $20^{\circ}$, respectively.

the experimental results, as shown in (figure 2(b)). For the SEM images at $\theta=0^{\circ}$, well separated nanotriangles with concave sides were formed. When $\theta$ increased to $10^{\circ}$, the nanopattern became separated, larger equilateral triangle. The high-magnification SEM images showed that the Ag nanopatterns also contain small Ag nanoparticles (NPs) formed around each apex of the triangle, with a solid hexagon-like pattern in the middle. For $\theta=15^{\circ}$, the resulting pattern emerged as an elongated triangle with a slight depression at its center, filled randomly with NPs. The neighboring nanopatterns were connected to one another at the respective apex of each triangle. When $\theta$ increased to $20^{\circ}$, the triangular nanopattern appeared wider, and consisted of different sized Ag NPs. The middle of each pattern had smaller and less dense AgNPs compared to the edges of the triangle.

Though the experimental results matched well with the NCs, there were morphology differences at $\theta>0^{\circ}$. The individual unit cell consisted of multiple Ag NPs, rather than the smooth, singular Ag patterns predicted in (figure 2(a)). The reason is that the NC used a continuous mathematical model, while during deposition, other growth related phenomenon such as adatom surface dynamics can take place. In particular, for low melting point $\left(T_{\mathrm{m}}\right)$ metals, like $\mathrm{Ag}$, not only is surface diffusion important, but the sticking coefficient during the initial nucleation of the vapor can also play a dominant role. For example, Kosiorek et al can match their experimental $\mathrm{Ni}$ and $\mathrm{Cr}$ SNSL nanopattern with their $\mathrm{NC}$ since both $\mathrm{Ni}$ and $\mathrm{Cr}$ have $T_{\mathrm{m}}=1453^{\circ} \mathrm{C}$ and $1857^{\circ} \mathrm{C}$, respectively [17]. We have also deposited $\mathrm{Ti}$ nanopatterns with $T_{\mathrm{m}}=1660^{\circ} \mathrm{C}$ at $\theta=15^{\circ}$ for comparison with those from $\mathrm{Ag}$ with $T_{\mathrm{m}}=961{ }^{\circ} \mathrm{C}$, see supplementary information S4. The resulting Ti nanopattern was smooth with no NPs in the unit cell as compared to those formed by Ag. Thus, to better reflect the formation of Ag nanopatterns, one has to consider the effect of surface diffusion and reemission.

The effects can only be accounted for in MC simulations. (Figure 2(c)) shows the simulated results using our MC deposition model. The overall patterns matched well with both the NC and experimental results. However, when $\theta \geqslant 10^{\circ}$, Ag NPs started to form within and around the patterns, especially when $\theta \geqslant 15^{\circ}$, the simulated results resembled more like the experimental results, which demonstrated that adatom surface dynamics did play an important role in SNSL pattern formation.

To quantitatively compare the nanopattern morphology from SEM, numerical simulation, and MC simulation, we define a geometric factor $\eta$, as the ratio of the in-plane height, $a$, and the length between opposing nanopatterns, $d$, i.e., $\eta=a / d$. Figure 3 shows the plot of $\eta$ versus $\theta$, and the inset SEM images are also shown in the figure to illustrate $d$ and $a$ measurements. We notice that, for $\theta=0^{\circ}$ the $\eta$ values for MC simulation and SEM matched more closely than that from NC. This is because the nanopatterns of both the MC and experiment had slightly rounded apex due to the shadowing effect of the PS nanosphere, therefore the $a$ value was slightly shorter than that of the NC. When $\theta=12^{\circ}$, the results from SEM and NC agreed more closely, and the thickness distribution of the pattern was greater towards the center of the 


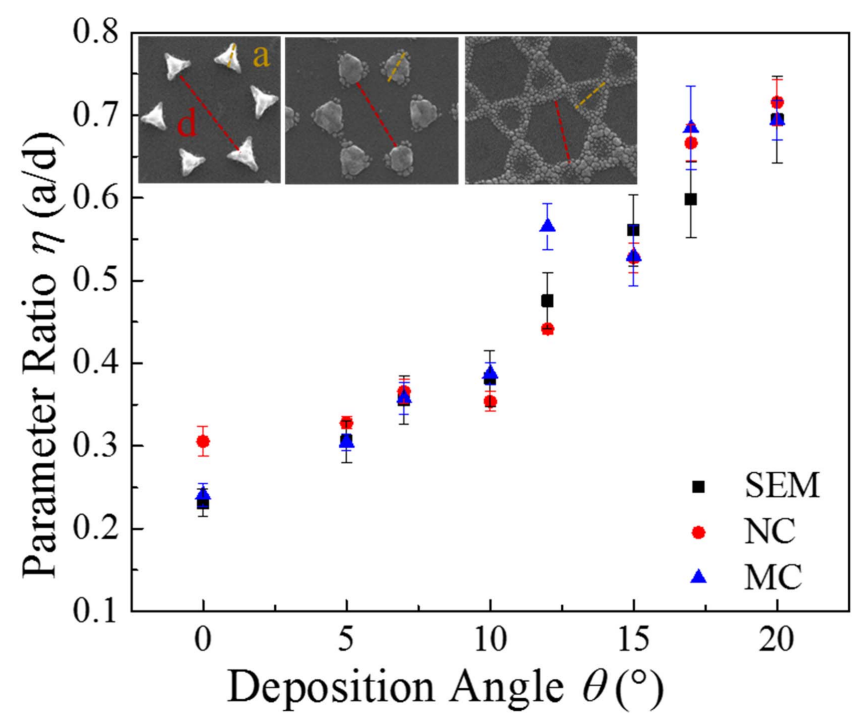

Figure 3. The shape parameter ratio $\eta$ as a function of $\theta$ obtained from the numerical calculations, SEM images, and MC simulations.

unit cell for the NC and SEM, while the MC predicted a more uniform distribution of Ag. For $\theta \geqslant 15^{\circ}$, the MC, SEM, and NC showed similar $\eta$ values. Thus, the MC simulation and $\mathrm{NC}$ both show that they can predict the shape of the Ag nanopatterns.

\section{Optical properties}

The extinction spectra of the Ag nanopatterns shown in (figure 4(a)) all exhibited multiple resonance peaks within the $300-1500 \mathrm{~nm}$ wavelength range. When $\theta<12^{\circ}$, all the samples had three resonant peaks, while when $\theta \geqslant 12^{\circ}$, there were only two peaks. The strongest extinction peak red shifted with $\theta$. (Figure 4(b)) plots resonance peak wavelengths $\lambda_{0}$ as a function of $\theta$. For $\theta=0^{\circ}$, three resonances occurred at $\lambda_{0}=363 \mathrm{~nm}, 423 \mathrm{~nm}$, and $689 \mathrm{~nm}$, respectively. These spectra features are typical for Ag nanotriangle arrays obtained from NSL $[29,30]$. The strong $\lambda_{0}=689 \mathrm{~nm}$ peak is due to a strong dipole resonance; the peak at $\lambda_{0}=423 \mathrm{~nm}$ is a weak in-plane quadrupole resonance, while the peak at $\lambda_{0}=325 \mathrm{~nm}$ is due to the out-of-plane quadrupole extinction $[29,30]$. When $\theta$ was increased to $5^{\circ}$ and $7^{\circ}$, the nanopatterns were still disconnected nanotriangles, and it was expected that the spectral features were similar to those of $\theta=0^{\circ}$ sample: the strongest resonant peak located at $\lambda_{0}=678-685 \mathrm{~nm}$, and two other peaks at $\sim 477$ and $\sim 347 \mathrm{~nm}$, which may all correspond to the dipole and quadrupole resonances as discussed for $\theta=0^{\circ}$ sample. However, the extinction peak became stronger, especially at $\lambda_{0}=678-685 \mathrm{~nm}$ region, and the corresponding peak width was smaller compared to that of $\theta=0^{\circ}$ sample. Such a narrow extinction peak is preferred for high sensitive LSPR sensors. When $\theta=10^{\circ}$, there were still three peaks observed and the peak at $\lambda_{0}=677 \mathrm{~nm}$ became even stronger. However, when $\theta$ was increased to $12^{\circ}$, the spectrum became very different: only two peaks were observed, with the strongest peak suddenly shifted to $\lambda_{0}=833 \mathrm{~nm}$, while the second peak $\left(\lambda_{0}=471 \mathrm{~nm}\right)$ stayed almost unchanged. With the further increase of $\theta$, the second peak location almost did not change while the largest peak kept on red shifting almost linearly with respect to $\theta$ until $\theta=20^{\circ}$. Such a change in the extinction spectra at $\theta \geqslant 12^{\circ}$ is consistent with the changes in nanopatterns. As shown in (figure 2(b)), when $\theta<12^{\circ}$, the nanopatterns were essentially a separated, solid triangle array. Only at slightly larger $\theta$, there were small NPs appeared around the solid triangles. However, when $\theta \geqslant 12^{\circ}$, the main solid triangles changed into small $\mathrm{Ag}$ particles, and the adjacent $\mathrm{Ag}$ patterns started to connect together to form a network structure. Since some Ag NPs were very closely spaced, it was expected that the electromagnetic coupling between small Ag NPs would play an important role for the optical properties.

To gain a better understanding of the optical properties, FDTD calculations were carried out based on the nanopatterns at $\theta=0^{\circ}, 10^{\circ}, 12^{\circ}, 17^{\circ}$, and $20^{\circ}$, respectively, and the resulting numerical extinction spectra, the experimental spectra, and an inset of the simulated unit cell of the nanopattern array were plotted together in (figure 5). For all of the calculations, the thickness of the Ag nanopatterns was fixed at $100 \mathrm{~nm}, 43 \mathrm{~nm}, 24 \mathrm{~nm}$, and $6 \mathrm{~nm}$, respectively. These height values were based upon AFM average height measurements shown in supplementary information S5. For $\theta=0^{\circ}$, the calculated extinction spectrum showed a prominent and sharp LSPR peak at $\lambda_{0}=664 \mathrm{~nm}$, and a secondary peak at $\lambda_{0}=493 \mathrm{~nm}$. Two weaker peaks at $\lambda_{0}=416 \mathrm{~nm}$ and $374 \mathrm{~nm}$ were also visible. These peak locations were close in value to the experimental peaks at $\lambda_{0}=688,433 \mathrm{~nm}$, and $339 \mathrm{~nm}$. However, compared to the experimental peak at $\lambda_{0}=688 \mathrm{~nm}$, the calculated peak was narrower, and had a higher extinction. These deviations are due to the statistic variation in the shape, size, and thickness of the triangles obtained experimentally. For $\theta=10^{\circ}$, the calculated extinction spectrum showed a similar, but broader peak at $\lambda_{0}=786 \mathrm{~nm}$, and secondary peaks at $\lambda_{0}=664 \mathrm{~nm}$ and $436 \mathrm{~nm}$. By comparison, the experimental LSPR peak at $\lambda_{0}=677 \mathrm{~nm}$ was also much narrower and blue-shifted compared to that of simulation which, may be caused by broad deviations in the experimental nanopatterns. The experiment and simulation spectra show much broader extinction when $\theta=12^{\circ}$. The simulated spectrum showed a broad extinction ranging between 350 and $1050 \mathrm{~nm}$, with the highest extinction value at $\lambda_{0} \sim 863 \mathrm{~nm}$. The experimental spectrum also showed a broad spectrum with $\lambda_{0}=794 \mathrm{~nm}$. For $\theta=17^{\circ}$ the calculated spectrum was broad, covering the $450-1400 \mathrm{~nm}$ wavelength region, which was qualitatively consistent with the experimental spectrum. For $\theta=20^{\circ}$, the simulated spectrum show a broad extinction value between 300 and $1500 \mathrm{~nm}$, with a maximum extinction value at $\lambda_{0}=410 \mathrm{~nm}$. Similarly, the experiment extinction spectrum also shows a broad peak, with $\lambda_{0}=452 \mathrm{~nm}$. Clearly, the simulated spectra qualitatively matched well with the experimental spectra.

The broadening of the extinction spectra at $\theta>10^{\circ}$ are due to the appearance of smaller Ag NPs of different sizes and orientations as well as the electromagnetic coupling among the particle assembles [31]. This can be clearly demonstrated 

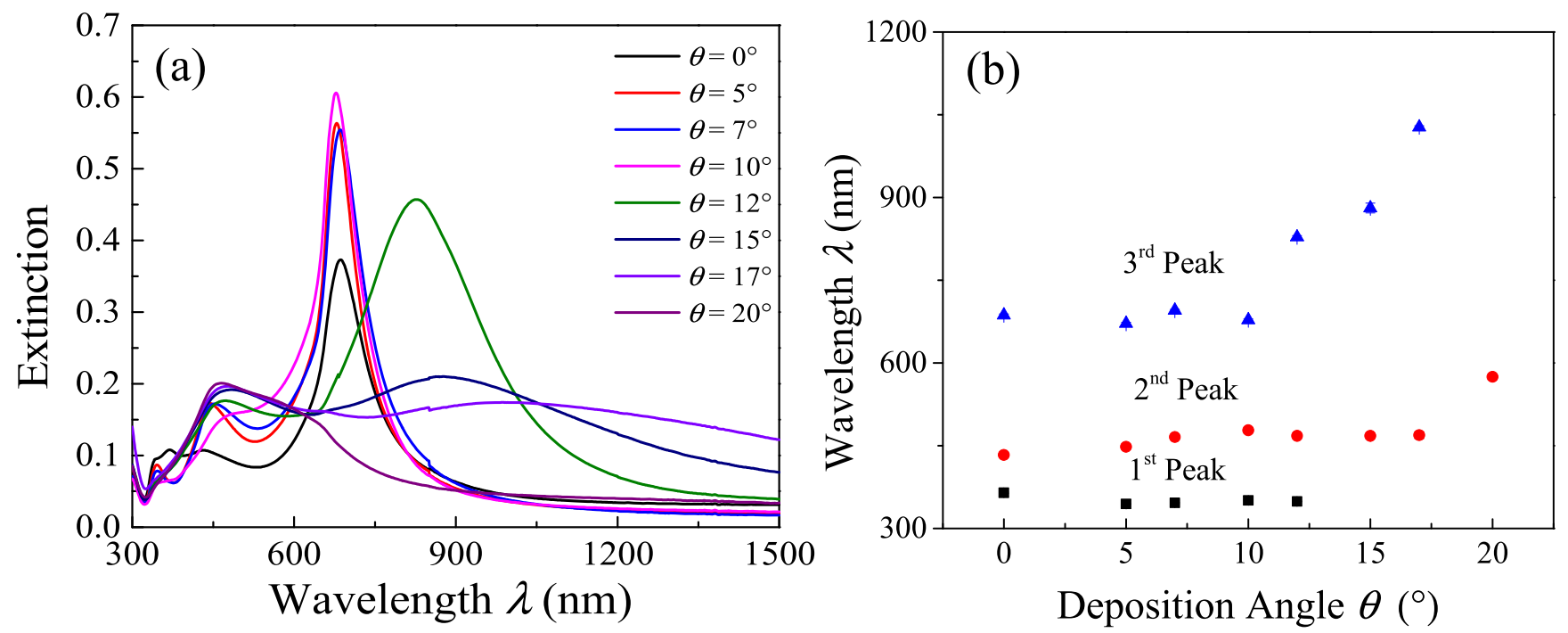

Figure 4. (a) Extinction spectra of SNSL nanopatterns at different incident angle $\theta$. (b) Extinction peak positions as a function of $\theta$.

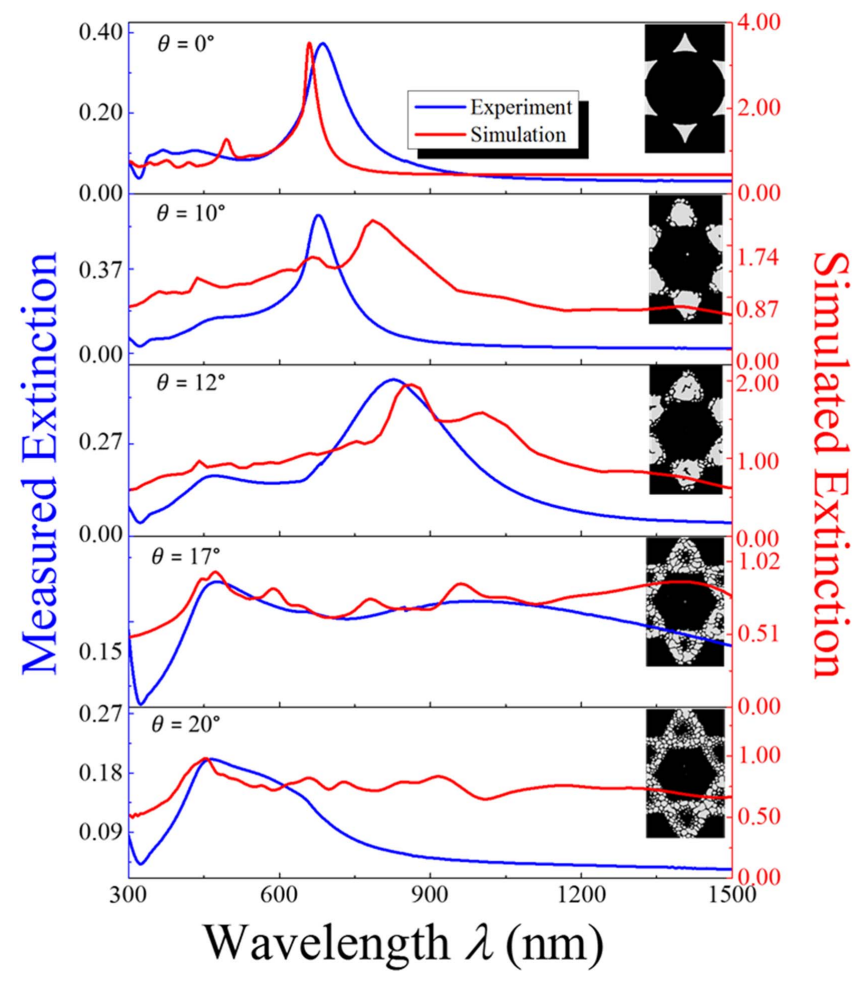

Figure 5. The comparison of the extinction spectra from finitedifference time-domain simulations and experimental results for selected $\theta$. The unit cell for FDTD simulation for each $\theta$ is shown as the insert image.

by investigating the polarization dependent extinction spectra as well as the local electric field distributions at the extinction resonant wavelengths. (Figure 6) shows an example for the Ag nanopatterns at $\theta=17^{\circ}$. The two orthogonal polarized extinction spectra, one excited by the horizontal $(x-)$ polarization, and the other by vertical ( $y$-) polarization, show distinguished different features: for $x$-polarization excitation, two strong resonances appeared at $\lambda_{0}=825$ and $1005 \mathrm{~nm}$, while under $y$-polarization, a strong resonant peak at $\lambda_{0}=684 \mathrm{~nm}$ appeared, though a relatively small resonant peak at $\lambda_{0}=1005 \mathrm{~nm}$ was still present. Such a difference originated from the orientation of the Ag NPs on the substrates, which means that the NPs are highly anisotropic. The multiple resonance features in both $x$ - and $y$-polarization excitations resulted from the size distribution of the Ag NPs. In addition, the local electric field distributions at the resonant wavelength shown in (figures 6(b) and (c)) demonstrated strong coupling among adjacent Ag NPs, especially large Ag NPs. For the $x$ polarization excitations at $\lambda_{0}=825$ and $1005 \mathrm{~nm}$, hot spots with strongest local electric field occurred only around largest Ag NPs due to their larger wavelengths; however, for the $y$ polarization excitation at $\lambda_{0}=684 \mathrm{~nm}$, hot spots occurred at multiple gaps among Ag NPs. Those high electric field hot spots were evidence of plasmonic coupling effect which would not occur for isolated nanopatterns formed at $\theta<10^{\circ}$. In addition, the high density of hot spots presented in (figure 6(d)) infer that those nanopatterns could be potentially used for SERS substrates.

\section{SERS activity}

Since the optical extinction of the $\mathrm{Ag}$ nanopatterns had a strong resonant peak at $\lambda_{0}=633 \mathrm{~nm}$ for samples at $\theta<12^{\circ}$, while for samples at $\theta \geqslant 12^{\circ}$, there were multiple hotspots available on the substrate due to the statistic arrangement of NPs in the pattern; it was expected that those patterned substrates will be good SERS substrates for excitation at $\lambda=633 \mathrm{~nm}$. (Figure 7(a)) shows the average BPE SERS spectra of different substrates. The characteristic peaks of BPE at $\Delta v=1200,1606$, and $1636 \mathrm{~cm}^{-1}$ were indicated in the figure, which corresponded to ethylenic $\mathrm{C}=\mathrm{C}$ stretching mode, pyridine ring $\mathrm{C}=\mathrm{C}$ stretching, and whole ring $\mathrm{C}=\mathrm{C}$ stretching mode respectively $[32,33]$. For $\theta=0^{\circ}$, there was no discernable BPE signal. For $\theta=5^{\circ}$, BPE peaks started to emerge, became stronger with the increase of $\theta$, and reached a maximum when $\theta=10^{\circ}$. With the further increase in $\theta$, all the BPE peaks decreased. In fact, the baseline corrected peak 

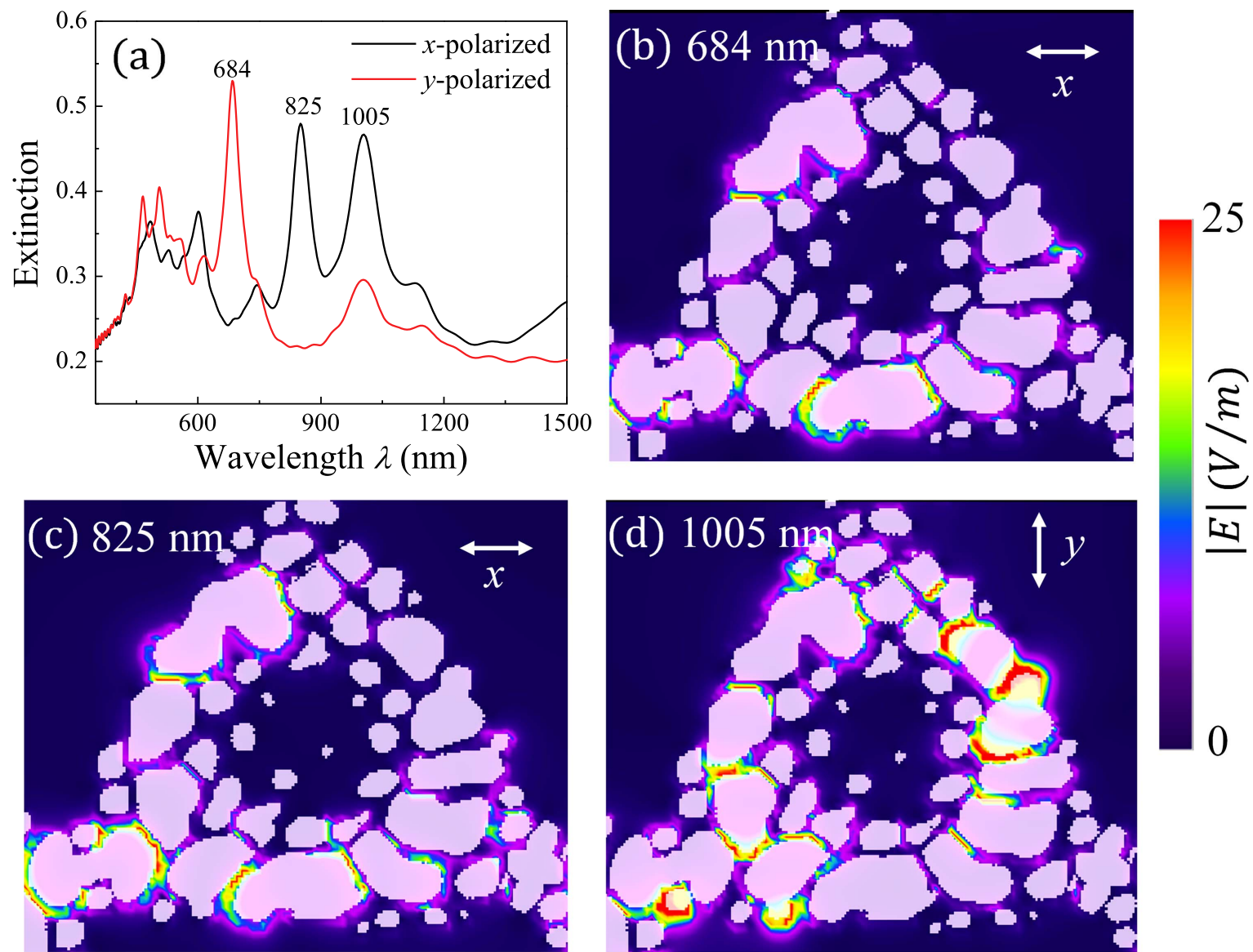

Figure 6. (a) Polarized extinction spectra of Ag nanopatterns at $\theta=17^{\circ}$; and the local electric field distributions of the $\theta=17^{\circ}$ nanopatterns excited by $x$-polarized light at (b) $\lambda_{0}=825 \mathrm{~nm}$ and (c) $\lambda_{0}=1005 \mathrm{~nm}$, as well as (d) $\lambda_{0}=684 \mathrm{~nm}$ excited by $y$-polarized light.
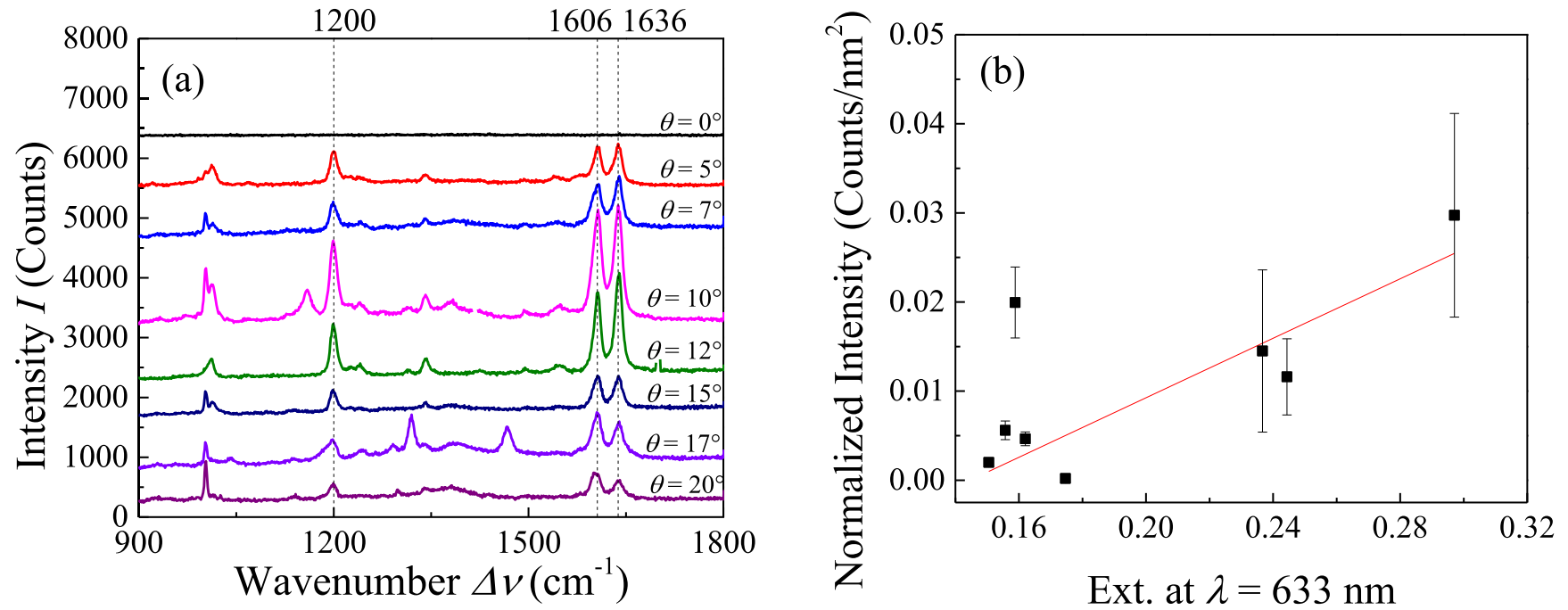

Figure 7. (a) BPE surface enhanced Raman scattering (SERS) spectra of SNSL Ag nanopatterns at different incident angle $\theta$. (b) Surface area normalized SERS peak intensity at $\Delta v=1200 \mathrm{~cm}^{-1}$ versus extinction spectra value at $\lambda=633 \mathrm{~nm}$.

height as a function of wavelength, shown in supplementary information S7, show quantitatively that for peak intensities at $\Delta v=1200,1606$, and $1636 \mathrm{~cm}^{-1}$, the greatest SERS intensity occurs at $\theta=10^{\circ}$.

However, since the nanopatterns were different at different incident angle $\theta$, the effective Ag surface area exposed to BPE was also different. If one assumes that the BPE were uniformly coated on the Ag surface, then different patterns would have different amount of BPE molecules coated on Ag surface, which could potentially give different strength of SERS signal. Therefore, to fairly compare the SERS response of different nanopatterns, the SERS intensity per unit Ag area 
needs to be considered. Based upon SEM micrographs and AFM height, the average surface area per unit cell of $\mathrm{Ag}$ nanopattern was calculated and the representative SERS peak intensity was normalized by the area. The area calculation and resulting normalized surface area for each deposition angle is shown in supplementary information S7. (Figure 7(b)) shows the result of the area normalized BPE peak intensity at $\Delta v=1200 \mathrm{~cm}^{-1}$ as a function of the optical extinction $\alpha$ at $\lambda=633 \mathrm{~nm}$. The normalized SERS intensity increased almost linearly with $\alpha$, which is consistent with other studies $[34,35]$. The substrate gives the highest SERS intensity is the nanopattern deposited at $\theta=10^{\circ}$. This result shows that using the SNSL one can tune the LSPR response and optimize SERS substrates.

\section{Conclusion}

In this work, we have investigated the morphological and optical properties of Ag nanopatterns prepared by SNSL. By changing the vapor deposition angle $\theta$, we showed that that a diverse variety of nanopatterns can be prepared. These patterns can be predicted by a numerical model as well as a MC simulation. Due to the variations on the size, shape, and arrangement of Ag NPs in the pattern, the optical properties of these nanopatterns can be tuned systematically, especially the LSPR wavelengths, and the results were confirmed by FDTD simulation. This is a relatively simple strategy to tune the LSPR based on NSL. We have demonstrated that the tuning of LSPR using SNSL nanopatterns can optimize the SERS response for chemical and biological applications.

\section{Acknowledgments}

WMD, YH, KS, and YZ are supported by National Science Foundation under Grant no. CMMI-1435309. DY is supported by National Science Foundation under Grant no. CHE1337700. WI thanks the support provided by the DOE Science Graduate Research Fellowship (SCGSR) fellowship and SLOAN fellowship. We also thank Dr Jason Locklin for the use of his AFM system for this project.

\section{References}

[1] Henzie J, Lee M H and Odom T W 2007 Multiscale patterning of plasmonic metamaterials Nat. Nanotechnol. 2 549-54

[2] Kabashin A, Evans P, Pastkovsky S, Hendren W, Wurtz G, Atkinson R, Pollard R, Podolskiy V and Zayats A 2009 Plasmonic nanorod metamaterials for biosensing Nat. Mater. 8 867-71

[3] Tsai Y-L, Chen T-G, Tsai M-A, Hsu C-W, Tseng P-C, Wang H-W, Han H-W, Jin L-H, Yu P and Shieh J-M 2011 Patterned glass substrates for enhanced solar energy harvesting in thin film solar cells 2011 37th IEEE Photovoltaic Specialists Conf. (PVSC) (Piscataway, NJ: IEEE) pp 945-7
[4] Hutter E and Fendler J H 2004 Exploitation of localized surface plasmon resonance Adv. Mater. 16 1685-706

[5] Mayer K M and Hafner J H 2011 Localized surface plasmon resonance sensors Chem. Rev. 111 3828-57

[6] Cao Z-S, Pan J, Chen Z, Zhan P, Min N-B and Wang Z-L 2011 Pure electric and pure magnetic resonances in near-infrared metal double-triangle metamaterial arrays Chin. Phys. Lett. 28057302

[7] Viarbitskaya S, Cuche A 1, Teulle A, Sharma J, Girard C, Arbouet A and Dujardin E 2015 Plasmonic hot printing in gold nanoprisms ACS Photon. 2 744-51

[8] Brolo A G 2012 Plasmonics for future biosensors Nat. Photon. 6 709-13

[9] Willets K A and Van Duyne R P 2007 Localized surface plasmon resonance spectroscopy and sensing Annu. Rev. Phys. Chem. 58 267-97

[10] Colson P, Henrist C and Cloots R 2013 Nanosphere lithography: a powerful method for the controlled manufacturing of nanomaterials J. Nanomater. 2013 $21-40$

[11] Santos A, Deen M J and Marsal L F 2015 Low-cost fabrication technologies for nanostructures: state-of-the-art and potential Nanotechnology 26042001

[12] Vogel N, Weiss C K and Landfester K 2012 From soft to hard: the generation of functional and complex colloidal monolayers for nanolithography Soft Matter 8 4044-61

[13] Zhang J H, Li Y F, Zhang X M and Yang B 2010 Colloidal self-assembly meets nanofabrication: from two-dimensional colloidal crystals to nanostructure arrays $A d v$. Mater. 22 4249-69

[14] Jensen T R, Malinsky M D, Haynes C L and Van Duyne R P 2000 Nanosphere lithography: tunable localized surface plasmon resonance spectra of silver nanoparticles $J$. Phys. Chem. B 104 10549-56

[15] Haynes C L and Van Duyne R P 2001 Nanosphere lithography: a versatile nanofabrication tool for studies of size-dependent nanoparticle optics J. Phys. Chem. B 105 5599-611

[16] Haynes C L, McFarland A D, Smith M T, Hulteen J C and Van Duyne R P 2002 Angle-resolved nanosphere lithography: manipulation of nanoparticle size, shape, and interparticle spacing J. Phys. Chem. B 106 1898-902

[17] Kosiorek A, Kandulski W, Chudzinski P, Kempa K and Giersig M 2004 Shadow nanosphere lithography: simulation and experiment Nano Lett. 4 1359-63

[18] Haynes C L and Van Duyne R P 2003 Dichroic optical properties of extended nanostructures fabricated using angleresolved nanosphere lithography Nano Lett. 3 939-43

[19] Kosiorek A, Kandulski W, Glaczynska H and Giersig M 2005 Fabrication of nanoscale rings, dots, and rods by combining shadow nanosphere lithography and annealed polystyrene nanosphere masks Small 1 439-44

[20] Gwinner M C, Koroknay E, Fu L, Patoka P, Kandulski W, Giersig M and Giessen H 2009 Periodic large-area metallic split-ring resonator metamaterial fabrication based on shadow nanosphere lithography Small 5 400-6

[21] Zhang G and Wang D Y 2008 Fabrication of heterogeneous binary arrays of nanoparticles via colloidal lithography J. Am. Chem. Soc. 130 5616-7

[22] Nemiroski A, Gonidec M, Fox J M, Jean-Remy P, Turnage E and Whitesides G M 2014 Engineering shadows to fabricate optical metasurfaces Acs Nano 8 11061-70

[23] Chen K, Rajeeva B B, Wu Z, Rukavina M, Dao T D, Ishii S, Aono M, Nagao T and Zheng Y 2015 Moiré nanosphere lithography ACS Nano 9 6031-40

[24] Ingram W M, Han C, Zhang Q and Zhao Y 2015 Optimization of Ag-coated polystyrene nanosphere substrates for quantitative surface-enhanced Raman spectroscopy analysis J. Phys. Chem. C 119 27639-48 
[25] Larsen G K, He Y Z, Ingram W and Zhao Y P 2013 Hidden chirality in superficially racemic patchy silver films Nano Lett. 13 6228-32

[26] Basnet $\mathrm{P}$ and Zhao Y 2016 Tuning the $\mathrm{Cu} \times \mathrm{O}$ nanorod composition for efficient visible light induced photocatalysis Catalysis Sci. Technol. 6 2228-38

[27] Taflove A and Hagness S C 1995 Computational electrodynamics: the finite-difference time-domain method Norwood 2nd edn (Boston, MA: Artech House Publishers) p 1995

[28] Yee K S 1966 Numerical solution of initial boundary value problems involving Maxwell's equations in isotropic media IEEE Trans. Antennas Propag. 14 302-7

[29] Jin R C, Cao Y W, Mirkin C A, Kelly K L, Schatz G C and Zheng J G 2001 Photoinduced conversion of silver nanospheres to nanoprisms Science 294 1901-3

[30] Sherry L J, Jin R C, Mirkin C A, Schatz G C and Van Duyne R P 2006 Localized surface plasmon resonance spectroscopy of single silver triangular nanoprisms Nano Lett. 6 2060-5
[31] Stranik O, Nooney R, McDonagh C and MacCraith B D 2007 Optimization of nanoparticle size for plasmonic enhancement of fluorescence Plasmonics 2 15-22

[32] Taylor C E, Garvey S D and Pemberton J E 1996 Carbon contamination at silver surfaces: surface preparation procedures evaluated by Raman spectroscopy and x-ray photoelectron spectroscopy Anal. Chem. 68 2401-8

[33] Yang W H, Hulteen J, Schatz G C and Van Duyne R P 1996 A surface-enhanced hyper-Raman and surface-enhanced Raman scattering study of trans-1, 2-bis (4-pyridyl) ethylene adsorbed onto silver film over nanosphere electrodes. Vibrational assignments: experiment and theory J. Chem. Phys. 104 4313-23

[34] Haynes C L and Van Duyne R P 2003 Plasmon-sampled surface-enhanced Raman excitation spectroscopy J. Phys. Chem. B 107 7426-33

[35] McFarland A D, Young M A, Dieringer J A and Van Duyne R P 2005 Wavelength-scanned surfaceenhanced Raman excitation spectroscopy J. Phys. Chem. B 109 11279-85 